\title{
Brevi considerazioni sulla familiarità perturbante di Dino Buzzati': Una animalesca metamorfosi terrificante
}

\author{
Elisa Martínez Garrido \\ Universidad Complutense de Madrid \\ elimarti@filol.ucm.es
}

\begin{abstract}
Il saggio studia il famoso racconto buzzatiano: I topi alla luce della psicoanalisi freudiana e dell'esperienza esistenziale dell'autore veneto, allo scopo di dimostrare la polisemica e ricca valenza ermeneutica dei roditori all'interno della narrazione. La presenza simbolica dei topi, legata a una chiara catena intertestuale, antica e moderna, struttura all'interno del rècit onorico di Buzzati, l'angoscia «edipica» di uno dei suoi testi più perturbanti.
\end{abstract}

Parole chiave: perturbante; angoscia; problematica edipica; topi, simbolo; Buzzati.

\section{Abstract}

This essays focuses on Buzzati's famous short story, I topi (Mice), in the light of Freudian psychoanalysis and the existential experience of this author of Veneto, in order to demonstrate the polysemous and rich hermeneutical representation of rodents in his narrative. The symbolic presence of mice, associated with a clear inter-textual chain, both ancient and modern, builds, through Buzzati's oneiric récit, the "oedipal» anguish of one of his most disquieting works.

Keywords: disquieting; anxiety; oedipal complex; mice; symbol; Buzzati.

«Il perturbante sarebbe quella sorta di spaventoso che ha a che vedere con le cose più conosciute e familiari, da tempo inmemoriale». ${ }^{2}$ Con queste parole, Freud nel suo saggio sul Perturbante ci allerta sulla profonda ambivalenza dell'aggettivo heimlich, perché se da una parte indica quello che è conosciuto

1. Questa parte del nostro lavoro prende ispirazione dal saggio di Graziella BERTO, Estranea familiarità, in Retoriche dell'alterità, "Aut, Aut», novembre-dicembre 1992, p. 47-55. Sul rapporto fra la letteratura fantastica e l'alterità cfr. Silvia ZANGRANDI, Il rapporto con il mondo autre, in Pagine infestate. I fantasmi e la tradizione fantastica del XX secolo, Milano: Arcipelago Edizioni, 2008, p. 41-66.

2. Cfr. Sigmund Freud, Lo siniestro (1919) in Obras completas, Madrid: Biblioteca Nueva, tomo VI, 1974, p. 2484. 
e familiare, dall'altra ha uno stretto rapporto con ciò che è occulto e nascosto. Non si deve dimenticare che il sostantivo Heim, casa, dimora, focolare, patria è alla base del nucleo semantico di heimlich, ma anche del suo antonimo unheimlich. Così la casa dell'uomo si trova sempre al posto dell' "Altro». ${ }^{3}$

Lo psicoanalista mette in contatto quello che è familiare e domestico con ciò che è più oscuro, inquietante e maligno; un rapporto in se stesso quasi magico, in cui il perturbate è ciò che vi è di più conosciuto e familiare (heimlich diventa unheimlich). Di conseguenza, "Unheimliche è tutto quanto sarebbe dovuto rimanere occulto, segreto, ma che invece si è manifestato». ${ }^{4}$ Il perturbante, quindi, comporta la «rivelazione» o la «manifestazione» di ciò che dovrebbe nascondersi, in un luogo in cui affiorano strane e inquietanti presenze che non avrebbero dovuto vedere la luce né avere spazio all'interno della domus. ${ }^{5}$ Pertanto, ciò che è per noi conosciuto e familiare, non è rassicurante quanto avevamo pensato. Siamo, insomma, davanti a un misterioso segreto che il fantastico e il perturbante mostrano in piena luce. ${ }^{6}$ Il perturbante, allora, non solo non ci allontana dalla nostra più familiare dimora, ma, al contrario, ci porta direttamente nei sotterranei più profondi ed oscuri delle sue viscere, dove vive accovacciato il mostro insondabile della famiglia. Per questo stesso motivo, l'unheimliche è sempre quello che ritorna, ciò che si ripete continuamente e incessantemente nel centro stesso del Heim.

La rivelazione di questa minaccia perturbatrice nascosta nelle profondità più intime della famiglia di Dino Buzzati, nella sua stessa casa d'infanzia, rappresenta il centro tematico di gran parte della sua letteratura fantastica ${ }^{7}$ e la casa di San Pellegrino, o le diverse varianti domestiche, ci mettono in rapporto diretto con il cuore stesso delle inquietudini inconscie dell'autore. Queste girano sempre intorno alla manifestazione delle diverse presenze perturbatrici dell'«a/Altro», diventando «reali» all'interno della domus, malgrado i suoi tentativi razionali per far sì che rimangano nascoste e i tanti animali

3. Cfr. Jacques LaCAN, Dal cosmo all'Unheimlichkeit, in Il seminario. Libro X. L'angoscia, Torino: Einaudi, 2007, p. 33-47.

4. Sigmund FreUd, Lo siniestro, cit, p. 2578.

5. Afferma Berto nel suo commento allo stesso saggio: "Unheimlich è un affiorare, piuttosto che un sottrarsi: ciò che ci turba è una presenza, piuttosto che una mancanza, qualcosa che si manifesta, uscendo dall'opacità in cui avrebbe dovuto rimanere...Una sfumatura distingue questi due modi dell'inquietudine, che tendono a confondersi: heimlich — nella sua seconda accezione, l'unica sopravvisuta - è la sensazione sottile di una presenza nascosta e indefinibile, che, pur molto vicina, agisce non vista (cfr. Il tramare alle spalle); unheimlich è invece lo svelarsi di tale presenza, nella sua inafferrabilità» (Graziella BERTO, cit, p. 53).

6. In questo senso sembra chiarificatore lo studio linguistico-etimologico di Gulio Lepschy sulla parola fantastico. Il linguista rivela il rapporto esistente fra il termine «fantastico» e la famiglia delle voci phantosomai, "farsi visible, apparire», da una parte, e la forma latina parlata senza aspirazione pantasia, con il significato di «visione», «angoscia», «incubo». Cfr. Gulio LEPSCHY, Aspetti linguistici del fantastico, in Nuovi saggi linguistici italiani, Bologna: il Mulino, 1989, p. 199-230.

7. Cfr. Stefano LAZZARIN, "Ces terrifiantes histoires de maisons hantées ...". Su alcune case infestate del Novecento italiano, in «Italianistica», n. 2-3, maggio-dicembre 2002, p. 163-180. 
inquietanti che vivono in segreto all'interno della casa, insieme ai rumori, agli spiriti ed ai fantasmi non sono altro che le multiformi traduzioni simboliche, ossessionanti, del suo stesso unheimliche.

Davanti a ciò che è perturbante, perché rimosso e negato, ma chiaramente certo, lo scrittore mostra la sua più che evidente ambivalenza, il fascino e il contemporaneo rifiuto verso le "visioni» interiori che vengono confessate attraverso la scrittura, nell'evidente duplicità dei suoi racconti, i quali girano sempre su ciò che gli è più familiare e amato: la casa, come rifugio e fortificazione contro il male, ma allo stesso tempo luogo che emana il più assoluto e temuto unheimliche. È l'autore stesso a confessarlo, quando dice che la casa

rappresenta uno dei fondamenti di quello che è il mio mondo poetico - $\mathrm{O}$ piuttosto di quello che ho dentro di poetico [...]. Questa casa è una cosa proprio fondamentale [...]. Il concetto di casa è per me quello di una fortezza domestica, entro la quale cercano di penetrare le sventure dal di fuori [...]. $\mathrm{Ma}$ indubbiamente nei bambini l'impressione che fanno le case è una cosa straordinaria...Gli schricchioli, la sera, la porta chiusa o aperta, di notte, nel buio [...]. Questo tipo di mistero - parlo del mistero della casa ma anche del mistero dei rumori del giardino- implica la presenza di entità sconosciute, probabilmente immaginarie... Spiriti, fantasmi, esseri della natura, elfi... Ecco: quelle cose lì... Chi è che passa nel corridoio, di notte? Sono i topi o è il vecchio nonno morto in peccato mortale?...E perché a dire il vero, il mistero della casa in fondo è più denso del bosco? Perché nella casa è abitata tanta gente. E questa gente ha lasciato — su questo c'è poco da discutere- qualche cosa nei muri. ${ }^{8}$

A partire da questa doppia prospettiva, bisogna leggere il racconto I topi, che fu pubblicato per la prima volta sul Corriere della Sera del 9 agosto 1953, l'anno successivo compreso nella raccolta $\mathrm{Il}$ crollo della Baliverna e poi nei Sessanta racconti del 1958. I topi può essere considerato uno dei testi più inquietanti della narrativa fantastica di Buzzati, dove si manifestano anche tutte le costanti strutturali, tematiche, simboliche e stilistiche della sua opera.

1. L' inversione e il capovolgimento dei principi e delle realtà del mondo naturale. In questo racconto i topi mangiano i gatti, schiavizzano e uccidono gli umani.

2. La mistura tematica fra la realtà e la fantasia, fra il vissuto, l'immaginato e il sognato.

3. La metamorfosi perturbante dell'animale semidomestico e inoffensivo (sorcio), che diventa, lungo lo sviluppo narrativo del testo, un mostro diabolico che si moltiplica.

4. Lo spostamento simbolico e connotativo dell'asse semantico spaziale su $v s$. giù. Nel racconto gli animali scendono dalla mansarda fino alla cantina.

8. Cfr. Yves Panafieu (a cura di), Dino Buzzati: un autoritratto, Milano: Mondadori, 1973, p. 12. 
Questa, come la fogna, ${ }^{9}$ è simbolo onirico delle parti «basse» del corpo umano. ${ }^{10}$

5. La condensazione e la rapidità del testo, costruito tramite la ripetizione (per cinque volte) dell'unico e stesso motivo narrativo, altamente perturbante, sempre in crescendo; che nel racconto è l'orrida presenza e moltiplicazione dei topi. In corrispondenza a questa, ogni volta che il protagonista-narratore rende conto all'amico-padrone di casa di tale fatto, si ripete da parte di quest'ultimo, la reiterata negazione (rimozione) dell'avvenimento angoscioso.

6. La ripetizione testuale nascosta dell'altra parola chiave del racconto: "paura», la quale agisce da leit motive perturbante nel momento centrale della storia. "Paura», con quattro occorrenze lessicali, diventa la chiave semantica del mistero inquietante di tutto il testo. La parola si trova al centro della confessione che Giorgio, il figlio più grande dei Corio, fa al narratore a proposito della paura del padre per i topi e della sua stessa incapacità di poter agire contro i terribili animali.

7. La ricorrente presenza simbolica e allegorica del male all'interno del testo, stabilita a partire dall'immaginario religioso cristiano di Buzzati. ${ }^{11} \mathrm{Il} \mathrm{rac-}$ conto traccia lo sviluppo tematico e narrativo della storia (come succede spesso all'interno dell'opera buzzatiana) attraverso lo scontro indiretto fra il Bene e il Male. Ne I topi, le bestie, alla fine del racconto, ormai diventate talpe dovoratrici, alludono indirettamente al bestiario infernale dantesco. ${ }^{12}$ La rivelazione e la scoperta dominatrice del male nel testo-casa-mondo, ci avvicina ancora, attraverso la condensazione allegorica presente nel simbolo dei topi e del loro dominio devastante sugli umani, al sentimento apocalittico di gran parte della narrativa buzzatiana. ${ }^{13}$

Come è stato già detto, la narrazione gira intorno al topos della "casa infestata»: una casa isolata in mezzo al bosco, nel limite fra lo spazio domestico e l'«a/Altro». Questa casa è un luogo familiare per l'io narrante (chiaro doppio di Buzzati) e ovviamente per i Corio, i proprietari. In essa il narratore-protagonista, trascorre le sue vacanze estive in loro compagnia, in pacifica beatitu-

9. Si ricordino anche Lettera noiosa de Le notti difficili (1971) o Eppure battono alla porta (pubblicato nel ' 40 per la prima volta) dei Sessanta racconti (1958), dove le parti basse della casa rappresentano i luoghi del mistero perturbante.

10. Rimandiamo alle rifessioni freudiane sulla simbologia sessuale della casa. Cfr. Sigmund FREUD, La representación simbólica en el sueño. Nuevos sueños típicos, in La interpretación de los sueños (1900), in Obras completas, cit., tomo II, 557.

11. Cfr. Sigmund Freud, El sueño de angustia, en Psicología de los procesos oníricos, en La interpretación de los sueños, cit., 693.

12. E giustamente la scelta della parola «rombo" quella che ci mette sulla possibile traccia dantesca nella scena finale del racconto studiato. Cfr. Nella GIANETTO, Sessanta racconti e una lingua da scoprire, in Il sudario delle caligini, Firenze: Leo Olschki, 1996, p. 209.

13. Si pensi, per esempio, a All'idrogeno e anche a Rigoletto, contenuti nei Sessanta racconti. Cfr. Dino Buzzati, Opere scelte, a cura di Lorenzo Carnazzi, Milano: Mondadori, «I Meridiani», 2002, p. 856-861 e 889-893. 
dine, ma la situazione muta quando inizia la narrazione di alcuni fatti estranei, in apparenza insignificanti, che costituiranno la base del racconto. Il récit, per questa ragione, si svolge in presente continuo e il narratore s'interroga, in modo breve e rapido, su ciò che sta accadendo nella Doganella, allo scopo di poter decifrare il mistero che ha reso impossibile il suo abituale ritorno in un posto per lui tanto familiare: "Che ne è degli amici Corio? Che sta accadendo nella loro vecchia villa di campagna, detta la Doganella?». ${ }^{14}$

Dopo l'incipit breve, quasi telegrafico, il narratore, ricorda i tempi felici trascorsi durante l'estate con gli amici nella casa di famiglia e introduce, quasi in modo involontario, di sfuggita, ciò che costituirà l'essenza della narrazione: l'elenco delle bizzarre memorie che cominciano a mostrarsi nella loro vera dimensione, e che, nell'affiorare, si rivelano in tutta la loro potenza terrificante: «Quanti giorni lieti ho vissuto in casa loro, nella solitudine dei boschi. Dai vecchi ricordi oggi per la prima volta affiorano dei piccoli fatti che allora mi parvero banali o indifferenti. E all'improvviso si rivelano». ${ }^{15}$ Questi ricordi servono da risposta alle domande precedenti e anche a quelle ultime con le quali si chiude la narrazione: «E adesso?, Perché Giovanni ha scritto di non poter più invitarmi? Cosa è successo?». ${ }^{16}$

La struttura ripetitiva e circolare di tali terribili domande, in stretto rapporto con l'animalesca invasione della casa degli amici, sempre in crescendo, prenderà nel racconto una progressione testuale minacciosa e perversa: il topolino fragile della prima vacanza si rivelerà alla fine della storia una presenza molto perturbante, la quale ha già a che vedere con il Male e con la sua paurosa metamorfosi. Ogni apparizione degli animali, separata tipograficamente dal punto a capo, e, di conseguenza, indipendente in rapporto alla manifestazione della sua progressiva testualità, comincia con un sintagma temporale: «Da un' estate lontanissima [...] parecchio prima della guerra [...]», «Passa un anno», «Passa un altro anno», «E l'anno dopo [...]», «Così per anni [...]». Fatto che evidenzia l'antica e ripetuta minaccia degli animali terribili all'interno della casa dei Corio, e la conseguente negazione da parte del proprietario. Si tratta indubbiamente di un'antica presenza, di un'inquietudine atemporale sempre in agguato nella casa.

Dopo i ricordi del passato, elencati dalla voce narrativa, siamo già in grado di intuire la dimensione profonda di quello che è «veramente» successo all'interno della casa di campagna. I topi, diventati mostri divoratori, mangiano i gatti di casa, uccidono i nonni e schiavizzano Elena, la madre della famiglia. Lei apparirà alla fine come una miserabile cenerentola, davanti a un grande pentolone, nel quale cucina per i suoi padroni. La casa di villeggiatura è diven-

14. Cfr. Dino Buzzati, I topi in Opere scelte, cit., p. 833. Forse sarebbe il caso di pensare alle valenze semantiche contenute nel nome della casa dei Corio: la Doganella, creato a partire dal sostantivo «dogana»: posto di frontiera fra paesi diversi dove si deve pagare per le merci che passano da una parte all'altra del territorio.

15. Il corsivo è nostro.

16. Cfr. Dino Buzzati, I topi, cit., 837. 
tata, quindi, un'altra casa tomada ${ }^{17}$ dalle forze strane e straniere, dalle bestie terrificanti e maligne che si sono impadronite della famiglia. Sono loro che adesso detengono un potere mostruosamente violento.

Anche se è vero che nella simbologia onirica c'è una differenza abissale fra un sorcio ed una talpa, dato che le valenze semantiche di questi «animaletti» sono ambivalenti, $i$ topi fin dall'antichità sono stati presenti nelle favole e nelle leggende popolari. ${ }^{18}$ La loro enorme e più mite popolarità, all'interno dell'immaginario fantastico occidentale contemporaneo, si è vista indubbiamente aumentata, però, grazie al cinema e soprattutto grazie all'industria Disney. Ma entrambi non hanno potuto mitigare completamente i loro aspetti più oscuri.

Dobbiamo ricordare che i roditori, e fondamentalmente le talpe, sono state nelle fiabe figure ambivalenti data la loro stessa familiarità perturbante. Perché se da una parte, i piccoli topi sono rappresentanti ingenui del mondo infantile, date le loro dimensioni, dall'altra simbolizzano anche l'eterno timore umano verso l'«a/Altro». Saccheggiatori del grano, propagatori delle malattie, divoratori dei piccoli esseri viventi, potentissimi riproduttori della loro specie, sono stati per gli umani un grave pericolo per la salute. Pertanto, rappresentano uno dei nostri nemici più inquietanti, e tante volte sono stati anche visti persino come un simbolo malefico.

D'altra parte, da una prospettiva inconscia, data la loro poderosissima capacità riproduttiva, i roditori sono associati direttamente alla sessualità, e spesso a una sessualità oscura e sadica. Da una prospettiva ermeneutica meno minacciosa, tenendo conto della debolezza e dell'ingenuità del sorcio, il topolino, nonostante il suo aspetto più simpatico e grazioso, continua ad essere un animale inquietante. In ragione della sua stessa piccolezza, ha la capacità di guardare tutto, di conoscere tutto. Il topo osserva di nascosto tutto quanto succede all'interno della casa e dal suo silenzioso nascondiglio è in grado di contemplare i misteri domestici proibiti, soprattutto durante la notte. Cioè, in un certo senso, il piccolo animale è il vero conoscitore della vita segreta della famiglia. Il suo atteggiamento ha, dunque, qualcosa del voyeur, e, contemporaneamente, data la sua stessa morfologia e il suo continuo andirivieni dalla propria tana,

17. Alludiamo al racconto di Julio Cortázar, Casa tomada, contenuto nel suo Bestiario (1951). Cfr. Julio CorTázar, Bestiario, Madrid: Alfaguara, 1961, p. 56-61. Come si ricorderà in questa narrazione una strana e perturbante presenza occupa la casa di una coppia di fratelli (uomo e donna) che vivono in perfetta pace ed armonia. Alla stessa raccolta dello scrittore argentino, appartiene anche Una señorita en París. In questo caso il fantastico perturnbante è simbolizzato tramite la riproduzione, non maligna, dei coniglietti che si molteplicano nella casa della protagonista (che è in viaggio a Parigi). Lei lascia in prestito l'appartamento di Buenos Aires a un suo amico, è lui che trova questi piccoli animaletti in ogni angolo della dimora. Entrambi i racconti di Cortázar parlano della sessualità negata, quella che si rivela trasversalmente tramite le diverse presente perturbanti dei due testi: l'incesto e la riproduzione. Cfr. Giovanna IOLI, Dino Buzzati, Milano: Mursia, 1988, cit., p. 33.

18. Si pensi per esempio a Il gatto e i topi di Esopo, a La battaglie dei topi e le donnole di Fedro o a I topi e il gufo de La Fontaine. 
può anche essere immaginato come un simbolo fallico della libido infantile. ${ }^{19}$ Per questo motivo, Buzzati, quando usa nel suo racconto il simbolo del topo, ci parla, tramite l'allegorismo del suo bestiario, della propria paura e della propria minacciosa angoscia sessuale (vicina e familiare), la quale cresce di nascosto all'interno della propria domus.

Dobbiamo anche tener conto che la stessa simbologia animale si inserisce all'interno di tutta una tradizione letteraria vicina a lui. I topi entrano, quindi, in diretto rapporto intertestuale con un insieme di opere dove i roditori sono elementi perturbanti, che ci portano verso una sessualità colpevole e mortale. Da Il pifferaio magico dei fratelli Grimm (autori tanto ammirati da Buzzati), ${ }^{20}$ a Schiaccianoci e il re dei topi di Hoffmann, ${ }^{21}$ Il pozzo e il pendolo di Poe, ${ }^{22}$ Il piccolo Eyolf di Ibsen ${ }^{23}$ o La peste di Camus, ${ }^{24}$ questi animali, fra il domestico e il selvatico, ci mettono sempre nella traccia della sessualità e della riproduzione, della colpa e della morte. Buzzati, che condivide con gli scrittori prima citati, tanto l'allegorismo della sua letteratura, quanto i suggerimenti emozionali propri dell'implicito, essendo anche lui un maestro del non detto, si serve delle potenti connotazioni simboliche, infantili e popolari, appartenenti al mondo dei roditori, con la finalità di confessare la sua angoscia infantile, in stretto rapporto alla problematica irrazionale del proprio vicenda sessuale.

All'interno di questa linea ermeneutica, bisogna anche ricordare che il simbolo dei topi si trova anche presente nel saggio freudiano Il caso clinico dell'uomo dei topi (1909), dove l'autore mette in evidenza lo stretto rapporto esistente fra i roditori, la sessualità sadica, la colpa e la nevrosi ossessiva. Anche Jung nella sua Metamorfosi e simboli della libido (1912) afferma che il simbolo animale è sempre una figura sessuale; un chiaro sostituto fallico saldamente legato alla problematica dell'incesto. ${ }^{25}$

Su queste basi non sembra inverosimile «sospettare» che il «sorcio minuscolo, grazioso e fragile» che apre il nostro racconto, entrando nella camera da letto del narratore-protagonista, ci metta già sulla strada della timida presenza

19. Cfr. Carl Jung, Metamorfosis y simbolos de la libido, en Gilbert Durand, Las estructuras antropológicas del imaginario, Madrid: Taurus, 1982, p. 67-69.

20. Cfr. Yves Panafieu, cit, p. 25.

21. Ibid., p. 25, 35, 175.

22. Anche Poe è alla base del fantastico buzzatiano e di tutto il genere. Cfr. Yves Panafieu, cit, p. $12,25,28,31,175$. Hoffmann e Poe sono indubbiamente i grandi maestri dello scrittore. Cfr. Nella GianetTo, Buzzati, Hoffmann e Poe, in Il sudario delle caligini, cit., p. 75-104.

23. Anche se non ci sono studi sulla traccia di Ibsen in Buzzati, pensiamo che è il sapore dei mondi del nord, inquietante e perturbante, ciò che ci consentirebbe di parlare dei possibili legami fra i due scrittori.

24. Si ricordi che l'inizio angosciante del romanzo di Camus è anche basato sulla perturbante presenza in crescendo delle talpe morte, annuncio di ciò che avverrà più tardi. Il rapporto fra Buzzati e Camus è molto conosciuto; l'adattamento francese di Un caso clinico si deve proprio a Camus. Cfr. Lorenzo Viganò, Album Buzzati, cit., Milano: Mondadori, 2006, p. $238-242$.

25. Siamo dell'avviso che sarebbe interessante studiare più approfonditamente il rapporto esistente fra l'animale totemico, i ratti divoratori e tutta la problemática sessuale dell'incesto. 
delle prime manifestazioni sessuali di un soggetto maschile (il nostro autore); cioè, davanti alla crescita della propria libido e alla potenza inarrestabile che questa prenderà più tardi, all'interno della propria famiglia e soprattutto in rapporto diretto alla figura della madre. La crescita e la moltiplicazione dell'animale rappresenterebbe, dunque, il passaggio dalla libido infantile alla sua posteriore fase adolescenziale. Tramite I topi, quindi, come in tanti altri casi, Buzzati confessa la sua stessa paura nei confronti alla propria sessualità. È questa la vera presenza perturbante del testo, quella che si è impossessata del suo desiderio irrazionalmente incestuoso. Assistiamo, nel racconto, quindi, alla progressiva trasformazione sessuale del soggetto maschile (Dino Buzzati) e al suo corrispondente terrore colpevole nei confronti di una tale realtà.

In questo modo ne $I$ topi, la mostruosa metamorfosi e riproduzione degli animali diventa un "frenetico brulichio di forme nere», "un laido tumulto", «una potenza infernale che nessuno avrebbe più fermato»; ${ }^{26}$ si arriva, così, fino alla visione malefica della propria crescita sessuale, in chiara rivalità con la figura del padre. I topi adulti annunciano, dunque, la potenza di una sessualità maschile matura, vista, però, come qualcosa terrificante alla luce edipica della colpa. Perciò non è casuale che sia Giorgio a raccontare al narratore le paure di suo padre nei confronti delle terribili bestie e dell'incapacità del genitore ad eliminarle.

Inoltre nel testo, insieme alla presenza del figlio adolescente e del suo «tradimento» del padre (in quanto rivale), c'è anche un'altra figura determinante per l'interpretazione del racconto nel senso incestuoso. Questa corrisponde al personaggio della madre-sposa e alla sua ultima immagine, con la quale si chiude il racconto.

Elena, serva dei topi, fa loro da mangiare. ${ }^{27} \mathrm{La}$ madre, «stanchissima e afflitta [...] cucina, rimestando in un immenso calderone, mentre intorno grappoli fetidi di topi la incitavano, avidi di cibo». ${ }^{28}$ La scena, vicina alla simbologia delle fiabe e dei sogni, presenta in se stessa una ricchissima polisemia che ci porta verso una catena di domande ermeneutiche senza risposta univoca. Nonostante tutte quante le possibili questioni interpretative poste dall'ultima immagine di Elena, queste continuano a metterci sulla strada percorsa finora, che progredisce intorno a costanti simboliche oniricamente incestuose.

Vediamo ora le domande «nascoste» più importanti, quelle che immediatamente ci pone il racconto.

1. Perché la madre del racconto rimane sola, come unica superstite di tutta la famiglia Corio?

26. Cfr. Dino Buzzati, I topi, cit., p. 838.

27. Secondo Freud, la cucina e le pentole ci mettono in contatto diretto con la genitalità femminile, cfr. Sigmund Freud, La interpretación de los sueños, cit., p. 575. Il fatto che Elena cucini davanti ad una grande pentola potrebbe rinforzare ulteriormente la prospettiva sessuale del racconto.

28. Cfr. Dino Buzzati, cit., p. 838. 
2. Perché, dice che è «troppo tardi» e che «non c'è più speranza»?29

3. I topi, dopo aver già mangiato i due grossi gatti della casa, hanno anche ucciso i nonni. Ma quale è stata la fine di Giovanni Corio e degli altri fratelli?

4. Che cosa sta cucinando Elena nella grande pentola?

5. I topi, diventati divoratori terribili, che cosa stanno mangiando? La carne di quelli che «sono stati fatti fuori» da loro stessi? La carne del padre morto?

Prima di tutto, dobbiamo tener presente che Elena (la madre del racconto) è l'unica che appare alla fine della "favola», perché il resto dei membri della famiglia, verosimilmente, sono stati eliminati dalle «bestiacce». Così scompare dalla storia fantastica di Buzzati qualsiasi possibile rivalità che possa intrommettersi fra la madre e il desiderio sessuale dei topi (cioè quello incestuoso del figlio).

In secondo luogo, è vero che il padre dei Corio era presente all'inizio della narrazione -in un primo momento l'amico ci parla di una lettera di Giovanni, dove gli si dice dell'impossibilità di trascorre le abituali vacanze estive nella loro casa di campagna_; ma è anche vero che, alla fine del testo, il padre non c'è più, è sparito, non c'è traccia di lui né degli altri membri della famiglia. Solo alla fine della narrazione, si sa qualcosa della devastazione della casa dei Corio e si scopre parzialmente il mistero che la avvolge, perché c'è una lontana voce corale che mormora. "Dicono" (il verbo è ripetuto per tre volte nel testo) che la casa è stata "occupata» da bestie mostruose. L'unico contadino che si è avvicinato al macabro luogo e che ha avuto la «visione infernale» di Elena Corio nelle circostanze già conosciute, le ha sentitito dire, come sappiamo già, che «è troppo tardi».

Ma perché è troppo tardi? Qual'è in realtà la sua situazione in mezzo ai topi affamati che la incitano a cucinare in continuazione? Anche in lei ha avuto luogo una metamorfosi, perché, essendo una donna «dolcissima e amabilissima» (possiamo dedurre che fosse anche bella), è diventata una "pezzente, [...] stanchissima ed afflitta» serva dei topi. Indubbiamente, la domanda centrale di tutto il nostro testo è questa: Elena li serve soltanto come cuoca? Dato lo stretto rapporto simbolico ed onirico fra la sessualità e il cibo, sembra abbastanza verosimile immaginare che le bestie terribili l'abbiano schiavizzata anche in senso sessuale. Per questo motivo, tutti gli altri umani/uomini della famiglia Corio sono «fuori» casa. I topi alla fine si sono così impossessati della madre e hanno, quindi, distrutto la Legge del padre e il tabù dell'incesto.

Alla domanda che riguarda il cibo cucinato da Elena per i voraci roditori, la risposta implicita può sembrare macabra, ma non inverosimile: la carne del padre, degli altri familiari uccisi. ${ }^{30}$ Questa possibilità sembra coerente anche con la simbologia degli animali divoratori dell'ultima parte del testo, in corri-

29. In realtà il testo dice "per noi non c'è più speranza».

30. Se ricordiamo la presenza dei roditori nel racconto di Poe prima citato, questa rinforza l'attendibilità della nostra analisi e la possibilità dell'antropofagia o il cannibalismo del testo. Le talpe de Il pozzo e il pendolo aspettano la morte dell' io-protagonista per soddisfare la loro voracità. 
spondenza al cannibalismo dei popoli primitivi e alla problematica edipica infantile, come riferisce Freud in Totem e tabù (1912-1913). ${ }^{31}$ Dobbiamo ricordare anche che l'antropofagia e il cannibalismo sono tematiche fortemente presenti in innumerevoli fiabe infantili: in Hansel e Gretel, in Pollicino o in alcune versioni de La bella addormentata, per esempio. In queste, come in tante altre favole, è adombrata la problematica dell'edipo, svelata dalle letture dell'antropologia e del folfklore. ${ }^{32}$ Non dobbiamo essere, dunque, colpiti da questa interpretazione, abbastanza familiare al mondo magico dei racconti popolari per l'infanzia. Per questo motivo, Elena, la madre del racconto, raddoppiando le sue caratteristiche di vittima in afflizione, appare alla fine del testo e dice al contadino che è «troppo tardi» e che «non c'è più speranza». Lei ormai è la doppia vittima di una sessualità golosamente $\mathrm{e}^{33}$ e mostruosamente incestuosa e violenta.

Ma bisogna anche dire che forse l'ultima immagine del testo non è tanto terribile come sembra. Non dobbiamo dimenticare che l'ironia parodica è sempre presente in gran parte dell'opera buzzatiana, e molto probabilmente è presente anche in questo caso, travestita nell'ultima «visione» de I topi. In un certo modo si potrebbe anche affermare che lo scrittore qui, come in tante altre occasioni, ci strizza l'occhio, ci fa un segno della sua trasversalità, ride dell'orrore eccessivamente visionario delle favole e dei propri incubi ${ }^{34}$ allo scopo di relativizzare e diminuire la paura che provoca in lui, forse, e nei lettori la schiavitù sessuale di Elena, preda e vittima dei terribili mostri incestuosi.

Buzzati ne I topi confessa, dunque, letterariamente e allegoricamente tutto quanto doveva rimanere taciuto, occulto, segreto, ma che, attraverso l'«incubo» del suo racconto, si è manifestato e rivelato. Siamo, dunque, davanti all'"affiorare» di quella presenza preturbante che non doveva aver spazio all'interno della domus, ma che malgrado tutto si è rivelata tramite la letteratura.

La narrazione buzzatiana, di conseguenza, testimonia l'insondabile mostro della sua/nostra immagine di famiglia. Perciò, i topi, chiara rappresentazione dell' unheimliche dello scrittore, sono la realtà che incessantemente si ripete al centro della sua casa. I topi rappresentano, di conseguenza, l'irrazionale e violenta forza sessuale dell'incesto e dell'edipo, quella che porta verso le più profonde viscere della dimora familiare: alla confessione perturbante dell'angoscia colpevole e alla morte, anche se questa, all'interno del proprio testo, è, contemporaneamente, relativizzata e "parodiata».

31. Cfr. Sigmund Freud, Totem y tabú (1912-1913), in Obras Completas, cit., tomo V, p. $1745-1850$.

32. Si ricordi nella stessa linea Vladimir Propp, Edipo alla luce del folklore, Torino: Einaudi, 1974.

33. L'orco delle fiabe ci mette in contatto diretto con lo sviluppo sessuale della prima fase orale. Cfr. Bruno BeTTELHeIM, Il mondo incantato: uso, importanza e significati psicoanalitici delle fiabe, Milano: Feltrinelli, 1977.

34. Forse nelle stesse parole di Elena: «è troppo tardi [...] per noi no c'è più speranza», tendenti in parte verso la tonalità melodrammatica, si camuffa l'écart che ci consente di parlare di parodia e di umore all'interno della visione infernale. La prima possibilità di lettura continua però ad essere, a nostro avviso, presente nel testo. 